\title{
NeuroImage
}

ELSEVIER

www.elsevier.com/locate/ynimg

NeuroImage 31 (2006) $1380-1388$

\section{The neural implementation of multi-attribute decision making: A parametric fMRI study with human subjects}

\author{
Stefan Zysset, ${ }^{*}$ Cornelia S. Wendt, Kirsten G. Volz, Jane Neumann, \\ Oswald Huber, and D. Yves von Cramon \\ Max Planck Institute for Human Cognitive and Brain Sciences, Stephanstrasse 1a, D-04103 Leipzig, Germany \\ University of Fribourg, Department of Psychology, Fribourg, Switzerland
}

Received 7 June 2005; revised 2 January 2006; accepted 12 January 2006

Available online 2 March 2006

\begin{abstract}
Decision making is not a unitary entity but involves rather a series of interdependent processes. Decisions entail a choice between two or more alternatives. Within the complex series of decisional processes, at least two levels can be differentiated: a first level of information integration (process level) and a second level of information interpretation (control level), leading to a subsequent motor response or cognitive process. The aim of this study was to investigate the neural network of these decisional processes. In a single trial fMRI study, we implemented a simple decision-making task, where subjects had to decide between two alternatives represented on five attributes. The similarity between the two alternatives was varied systematically in order to achieve a parametric variation of decisional effort. For easy trials, the two alternatives differed significantly in several attributes, whereas for difficult trials, the two alternatives differed only in small details. The results show a distributed neural network related to decisional effort. By means of time course analysis different subprocesses within this network could be differentiated: regions subserving the integration of the presented information (premotor areas and superior parietal lobe) and regions subserving the interpretation of this information (frontolateral and frontomedial cortex, anterior insula, and caudate) as well as a region in the inferior frontal junction updating task rules.
\end{abstract}

(C) 2006 Elsevier Inc. All rights reserved.

Keywords: Decision making; fMRI; Frontolateral cortex; Parametric modulation; Two-level model

\section{Introduction}

A decision arises when a person is consciously aware of two or more possible alternative behaviors (be it thoughts or actions), only

\footnotetext{
* Corresponding author. Max Planck Institute for Human Cognitive and Brain Sciences, Stephanstrasse 1a, D-04103 Leipzig, Germany. Fax: +49 3419940221.

E-mail address: zysset@cbs.mpg.de (S. Zysset).

Available online on ScienceDirect (www.sciencedirect.com).
}

one of which can or should be performed. This person (the decision maker) is thus forced to choose one option out of a set of alternatives. The process of making a decision is a sequence of subprocesses, e.g., evaluating specific aspects of each alternative, constructing a mental representation of the decision situation, or judging the involved uncertainty (Paulus et al., 2005). These subprocesses are at least to some degree interdependent. Most imaging studies investigating decision making have focused on subprocesses, like uncertain decision processes (Blackwood et al., 2004; Paulus et al., 2001; Volz et al., 2003), reward (Haruno et al., 2004; Rogers et al., 2004; Bush et al., 2002; Bechara et al., 1996, 1997, 2000), risk taking (Paulus et al., 2003a,b), ethical decision making (Heekeren et al., 2003), moral judgments (Heekeren et al., 2005; Moll et al., 2005), economic decisions (Sanfey et al., 2003), monetary gains (Gehring and Willoughby, 2002), or personal choice (Turk et al., 2004). An overview article by Krawczyk (2002) on the neural basis of human decision making shows that most research is done on the specific aspect of reward/punishment, emotion, and environmental adaptiveness. Very few functional imaging studies have investigated decision making as the process of relating several independent sources of variance, i.e., in the context of relational integration.

Functional magnetic resonance imaging (fMRI) studies have used mainly gambling situations as decision tasks. As recent research indicates, gambles omit relevant aspects of real-life decision making, such as active risk management (Huber, 2002). In order to get a better understanding of the complex neural network of human decisional processes, one should meet two goals: (i) the experimental situation ought to resemble real-life decision tasks (Ford et al., 1989); (ii) decision making should be stripped down to its bare essentials (Shadlen and Newsome, 1996).

The present study is a first attempt with a basic multi-attribute decision task, in which alternatives are evaluated according to several attributes. Uncertainty and risk could be introduced into a multi-attribute task, but in its basic version, these aspects are not involved. A typical example of such a multi-attribute task is the decision situation of a person who wants to rent an apartment. The 
decision maker may take into account the location, the quietness, the number of rooms, how luxurious the apartment is, the distance to the person's working place, whether there are shops and restaurants nearby, etc. Different attributes usually carry different importance or weight. If our decision maker is light sleeper, quietness of the apartment would get a high weight, if not, quietness may get only a small weight.

A multi-attribute decision situation can be represented by an Alternative by Attribute matrix, with alternatives as columns and attributes as rows. Table 1 shows the general scheme of such a multi-attribute task as used in our study. In making a decision, the decision maker usually combines her or his evaluations of the aspects and the weights of the attributes to an overall decision. Several theories have been developed to describe multi-attribute decisions, for example, the additive utility model (Slovic et al., 1977), simple heuristics (Payne et al., 1993; Rieskamp and Hoffrage, 1999; Svenson, 1979), or elementary information processing operators (Huber, 1989; Payne et al., 1993). In the present study, details of these theoretical approaches are not relevant.

We make the general assumption that the decision process consists of the application of one or more decision heuristics (e.g., Lexicographic heuristic, Weighted Pros, cf. e.g., Huber, 1989; Rieskamp and Hofrage, 1999; Svenson, 1979). A decision heuristic consists of a sequence of subprocedures that can be modeled as elementary cognitive operators. For example, the Lexicographic Heuristic consists of the following subprocedures (for a more detailed description in terms of elementary cognitive operators, see Huber, 1989): (i) weighting the attributes and selecting the most important one; (ii) evaluating and comparing the alternatives on this most important attribute; (iii) choosing the alternative that is better; and (iv) if no alternative is better, eliminating the most important attribute and restarting at item (i).

Cognitive process models of decision making, involving heuristics or elementary information processing operators, postulate at least two levels (or components) of the process: a process level and a control level. We regard the distinction of these two levels as being essential for the way decision making is reflected in the brain's activation pattern.

\section{Process level}

At this level, the subprocedures of the heuristic are performed, for example: evaluation of the alternatives features,

Table 1

Examples of two multi-dimensional decision task as used in the present study

\begin{tabular}{|c|c|c|c|c|c|}
\hline \multirow[t]{2}{*}{ Attribute } & \multirow[t]{2}{*}{ Renting a flat } & \multicolumn{2}{|c|}{ Easy task } & \multicolumn{2}{|c|}{ Difficult task } \\
\hline & & Alt A & Alt B & Alt $\mathrm{A}^{\prime}$ & Alt $\mathrm{B}^{\prime}$ \\
\hline 1 & Price square meter $\left(\right.$ Euro $\left./ \mathrm{m}^{2}\right)$ & 8 & 6.1 & 8 & 8.1 \\
\hline 2 & Size $\left(\right.$ in $\mathrm{m}^{2}$ ) & 59 & 90 & 72 & 69 \\
\hline 3 & $\begin{array}{l}\text { Distance to City Center } \\
\text { (minutes to walk) }\end{array}$ & 30 & 11 & 20 & 21 \\
\hline 4 & $\begin{array}{l}\text { Distance to public } \\
\text { transport (m) }\end{array}$ & 310 & 300 & 450 & 155 \\
\hline 5 & Distance to work $(\mathrm{km})$ & 5.1 & 1.0 & 2 & 1.9 \\
\hline
\end{tabular}

In each task, two alternatives are described on five attributes. It is assumed that a lower price, the larger size, and the shorter distances are preferred. In the easy task, alternatives A and B are dissimilar, whereas in the difficult task, alternatives $\mathrm{A}^{\prime}$ and $\mathrm{B}^{\prime}$ are similar. the weighting of attributes, but also the concatenation of aspects (e.g., the amount of rent and the extras can be concatenated as 'costs') or the trade-off of aspects (e.g., the higher rent of apartment $\mathrm{A}$ is compensated for by its better location). At this level, information search is also performed (e.g., what is the size of apartment A).

Let us consider the involvement of the process level, when the decision maker gets information about both alternatives sequentially, for one attribute after the other. Thus, the decision maker would acquire first information concerning aspects A1 and B1 (see Table 1), next about aspects $\mathrm{A} 2$ and $\mathrm{B} 2$, and so on. The subprocedures on the process level have to be activated for every presented attribute, starting with the first two aspects till the whole information is displayed.

\section{Control level}

This level constitutes a meta-level for the decision process. It governs the activities on the process level. It selects a decision heuristic and coordinates the subprocedures on the process level when performing a heuristic. The selected heuristic determines how the evaluations on different attributes are integrated. In the Weighted-Pros heuristic, for example, the weights of the attributes are relevant in the integration. The integration of the weights of different attributes is performed by a subprocess within the heuristic. The control level decides whether the alternative favored at the moment is distinctly better than the others (e.g., Svenson, 1993) and thus whether the decision process can be terminated or has to be continued. For example, in a situation with several alternatives, first a heuristic is used that enables a fast reduction of the set of alternatives by inspecting only one or two attributes (e.g., lexicographic heuristic). When the set of alternatives has been reduced to a short list, these alternatives are inspected in more detail with the help, for example, of the 'Weighted-Pros' heuristic. There are different assumptions about the control level, which the present study will not dwell upon any further (Huber, 1989; Payne et al., 1993; Svenson, 1993).

With respect to the temporal dynamics, the processes on the control level should behave differently than the process level: in the first information acquisition step, the control process should only be activated weakly if at all because the decision maker knows that more information is coming and that a final decision would be premature. Furthermore, after inspecting the alternatives on the first dimension(s), it may not yet be clear what heuristic is best. For example, use of the 'Weighted Pros' Heuristic (e.g., alternative A is better on dimension 1 and 2, but B is better on dimension 3) cannot be considered until a preference conflict occurs. Furthermore, a trade-off between dimensions cannot occur if only one dimension has been inspected. Over time, with the presentation of additional information, activation of the control process should increase until a decision is finally reached.

Because of this, different temporal dynamics of the BOLD response are expected in relation to the underlying processes: areas related to the process level should show an early onset of the hemodynamic response, with the BOLD signal steadily increasing. On the other hand, areas related to the control level should show a delayed signal increase. Further, response-related areas should show signal changes only at the time the response is given.

We additionally hypothesize that the amount of neural activation of the process level and control level should be related to the difficulty of the decision task. Difficulty in the present study 
is modulated via the similarity between alternatives: the more similar the alternatives are, the more difficult it becomes to decide between alternatives. It has been argued that similarity is the primary determinant of the degree of difficulty associated with decision making (Payne et al., 1993; Krawczyk, 2002). Consider two decision situations, where the information in five attributes is presented to the decision maker. In situation (a), alternative A is distinctly better than alternative $B$ in each of the attributes. In this case, the control process can is taxed minimally, as the final choice is obvious and remained unchallenged during the decision process. Consider, in contrast, situation (b): here, in attribute D1, alternative A is slightly better than B, in attribute D2, B is slightly better, in attribute D3 both alternatives are equally good, in attribute D4 alternative B is slightly better than A, and in attribute D5, A is slightly better than B. In this case, we expect a more elaborated control process because none of the alternatives is unambiguously better. The control process may have to meticulously compare the attributes and their weights or come to the conclusion that more information is needed. Thus, the more difficult a decision process is, the greater the anticipated involvement of the process and control levels will be. The measured BOLD response should differentiate between the levels of similarity.

A comparable two-stage model for visual discrimination in the rhesus monkey has been put forward by Shadlen and Newsome (1996, 2001). (For an overview of an animal decision-making model, see Reddi, 2001; Schall, 2002; Sakagami and Tsutsui, 1999; Kim and Shadlen, 1999). A recent fMRI study by Heekeren et al. (2004) showed that this two-stage model also holds for perceptual decision making in human subjects. The superior parietal lobe, related to the integration of information (Prabhakaran et al., 2000) and premotor structures (Heekeren et al., 2004) are hypothesized to be involved to the process level of decision making.

Several decision-making studies have described an involvement of the dorsolateral prefrontal cortex (DLPFC) in processing similarities and relational processing in decision making (Prabhakaran et al., 1997). Inductive, open-ended processing appears to be right lateralized, while explicit rule-based processing tends to be left lateralized (Goel and Dolan, 2000; Elliott and Dolan, 1998). As the present task is mainly based on explicit contextual cues, a left lateralization in the DLPFC activation is expected. Patient studies showed that lesions in the prefrontal cortex result in planning deficits (Goel et al., 1997). We hypothesize that regions in the prefrontal cortex are related to the control level of decision making.

The present study aims at localizing those neural structures involved in a more complex decision-making paradigm. We implemented a simple multi-attribute decision-making task (see Table 1), where subjects had to choose between two alternatives described on five attributes. The similarity between the two alternatives was varied systematically in order to achieve a parametric variation of decisional effort. For easy trials, the two alternatives differed significantly in several attributes whereas for difficult trials, the two alternatives differed only in small details. As mentioned above, for very different alternatives, the choice is obvious, reliable, and fast. If the similarity of the alternatives is high, the decision process becomes more difficult and is suggested to take longer. Along with the changing onset of the hemodynamic signal, this design allows to distinguish between areas involved in the process level and the control level of decision making.

\section{Methods}

Subjects

15 neurologically healthy subjects ( 7 female; mean age 26.6 years) were tested. Written consent from all subjects was obtained prior to the scanning session. All subjects had normal or correctedto-normal vision and were native German speakers. None of the subjects was taking medication at the time of the study. Subjects were instructed prior to the actual experimental session. They were given time to practice the task with its various conditions and the key allocations. Once they felt comfortable with the task, subjects were positioned supine in the scanner.

\section{Task and paradigm}

The subject's task was to decide between two alternatives described on five attributes (see Fig. 1). The alternatives were presented in a $2 \times 5$ matrix. Four different topics (along with their attributes) were used (see Table 2), all representing a familiar decision setting for the subjects. The presentation order of the attributes corresponded to their judged importance in order to simulate the course of a natural decision situation (Aschenbrenner et al., 1984). The attributes were always presented in the same order beginning with the most important attribute.

At the beginning of each trial, a matrix was shown with the generic terms of the relevant attributes, and the values of the first attribute for the two alternatives were filled in. Every $3 \mathrm{~s}$, the value of one additional attribute was added consecutively. The information remained on the screen until the end of each trial in order to minimize working memory load. After $12 \mathrm{~s}$, when all the values had been shown, participants gave their choice for one of the two alternatives. Subjects used their index and middle finger of the right hand to indicate which of the two alternatives they preferred.

The independent variable was the similarity of the two alternatives. In pilot studies, the importance of the attributes, as well as each individual value for each attribute, were rated. Based on the results of the pilot studies, pairs of alternatives were generated on the basis of the criterion-dependent-choice model (Aschenbrenner et al., 1984). In all trials, the two alternatives differed only marginally on the first attribute. Thus, a choice after

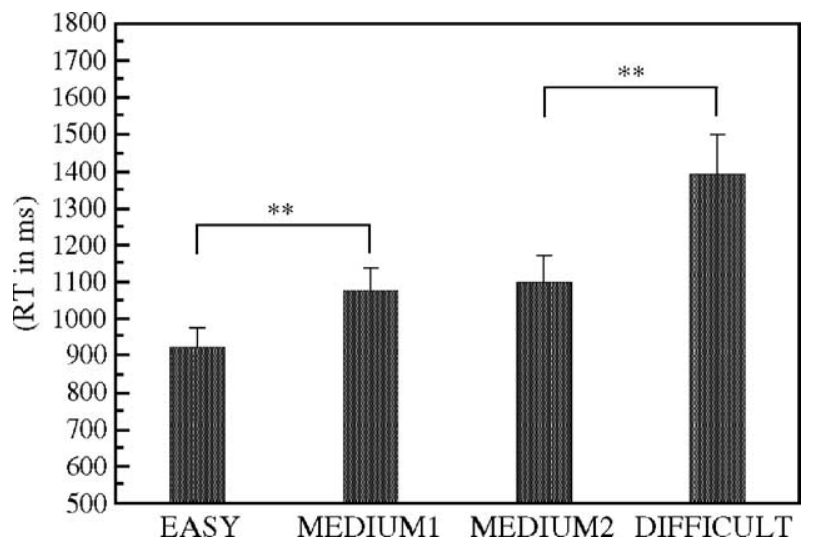

Fig. 1. Mean reaction times for the four similarity levels (Easy, Medium1, Medium2, Difficult) of the decisions averaged across all subjects and the different settings. Reaction times increase with decreasing differences between the two alternatives. ${ }^{* *}$ Indicates a $P$ value of $P \leq 0.001$. 
Table 2

Four topics described on five attributes were used in the present study

\begin{tabular}{ll}
\hline Topic & 5 Attributes \\
\hline Renting a flat & $\begin{array}{l}\text { Price per square meter, size, } \\
\text { distance to city center, distance } \\
\text { to public transport, distance to work }\end{array}$ \\
$\begin{array}{l}\text { Buying a } \\
\text { mobile phone }\end{array}$ & $\begin{array}{l}\text { rating by experts, size, weight } \\
\text { Buying a car }\end{array}$ \\
$\begin{array}{l}\text { Price, average petrol consumption, } \\
\text { annual car tax, technical test (TUV), mileage }\end{array}$ \\
Contract for a & Fee per month, call-charge per minute, \\
mobile phone & amount of free minutes, duration of \\
& contract, price for contract
\end{tabular}

These topics were rated in pre-tests by potential subjects as highly familiar situations. The attributes rated as the most important aspects for the specific topic were chosen for the experimental setting.

the presentation of the first attribute was not possible. In order to achieve a parametric variation of difficulty, the trials were assigned to four groups, based on the mean difficulty judgments of the pilot studies (25, percentile-splitting). For the easy decisions (level 1), the difference between alternatives was large, and a decision in favor of one alternative could be made after the presentation of the second or third attribute. For decisions with similarity level 2 (medium 1), the alternatives differed clearly, but only on attribute four and five. For level 3 (medium 2), the differences on attribute four and five were smaller. For difficult decisions (level 4), the differences were small on all attributes. By this, four different groups of trials with different similarity scores were generated. We did not generate trials with conflicting alternatives, where for example alternative A was clearly better on attribute 2 and alternative B was clearly superior on attribute 3 .

Each trial lasted $18 \mathrm{~s}$. Four trials were presented for each combination of the four level of similarity and four topics (16 trials for each level of similarity, 16 trials of each topic), resulting in 64 trials were presented. 8 additional resting baselines of $18 \mathrm{~s}$ were randomly introduced. A total number of 72 trials (21 min and $36 \mathrm{~s}$ ) were presented.

\section{MRI scanning procedure}

The experiment was carried out on a $3 \mathrm{~T}$ scanner (Siemens TRIO, Erlangen, Germany). 22 axial slices (19.2 cm FOV, 64 by 64 matrix, $4 \mathrm{~mm}$ thickness, $1 \mathrm{~mm}$ spacing), parallel to the AC-PC plane and covering the whole brain were acquired using a single shot, gradient recalled EPI sequence (TR $2000 \mathrm{~ms}$, TE $30 \mathrm{~ms}, 90^{\circ}$ flip angle). One functional run with 651 repetitions (648 time point for the presentation of the task +3 time point at the end) was run, with each time point sampling over the 22 slices. Prior to the functional runs, 22 anatomical T1-weighted MDEFT (Ugurbil et al., 1993; Norris, 2000) images (data matrix $256 \times 256$, TR $1.3 \mathrm{~s}$, TE $10 \mathrm{~ms}$ ) and $22 \mathrm{~T} 1$-weighted EPI images with the same spatial orientation as the functional data were acquired.

\section{fMRI data analysis}

The fMRI data were processed with LIPSIA software (Lohmann et al., 2001). This software package contains tools for preprocessing, registration, statistical evaluation, and presentation of fMRI data. Functional data were motion-corrected offline with the Siemens motion correction protocol (Siemens, Erlangen, Germany). To correct for the temporal offset between the slices acquired in one scan, a cubic-spline interpolation was applied. A temporal highpass filter with a cutoff frequency of $1 / 144 \mathrm{~Hz}$ was used for baseline correction of the signal, and a spatial Gaussian filter with $5.65 \mathrm{~mm}$ FWHM was applied.

To align the functional data slices onto a $3 \mathrm{D}$ stereotactic coordinate reference system, a rigid linear registration with six degrees of freedom (3 rotational, 3 translational) was performed. The rotational and translational parameters were acquired on the basis of the MDEFT and EPI-T1 slices to achieve an optimal match between these slices and the individual 3D reference data set. This 3D reference data set had been acquired for each subject during a previous scanning session. The MDEFT volume data set with 160 slices and $1 \mathrm{~mm}$ slice thickness was standardized to the Talairach stereotactic space (Talairach and Tournoux, 1988). The same rotational and translational parameters were normalized, i.e., transformed by linear scaling to a standard size. The resulting parameters were then used to transform the functional slices using trilinear interpolation, so that the resulting functional slices were aligned with the stereotactic coordinate system.

The statistical evaluation was based on a least squares estimation using the general linear model for serially autocorrelated observations (see also Friston et al., 1995; Worsley and Friston, 1995; Aguirre et al., 1997; Zarahn et al., 1997). The design matrix was generated from a box-car function, convolved with a hemodynamic response function (constructed by a gamma density function; Glover, 1999), including the individual trials and the resting baseline. An additional parameter for each trial was included, representing the similarity level of each trial. For each level, the mean reaction time (in seconds) of that level was used as parameter. The model equation, including the observation data, the design matrix and the error term, was convolved with a Gaussian kernel of dispersion of $4 \mathrm{~s}$ FWHM to account for the temporal autocorrelation (Worsley and Friston, 1995). In the following, beta-values were estimated. As the individual functional datasets were all aligned to the same stereotactic reference space, the single-participant contrast images were then entered into a second-level random effects analysis for each of the contrasts. The group analysis consisted of a onesample $t$ test across the contrast images of all subjects that indicated whether observed differences were significantly distinct from zero (Holmes and Friston, 1998). Subsequently, $t$ values were transformed into $Z$ scores. We corrected $P$ values for multiple comparison using the False Discovery Rate method (Genovese et al., 2002; Benjamini and Hochberg, 1995; Benjamini and Yekutieli, 2001) with at least $P<0.01$.

Further, a time course analysis of the fMRI signal was calculated. Trial-averaged time courses (stimulus onset locked) for the resting baseline and the task conditions were extracted from the preprocessed data on a voxel-by-voxel basis for each subject at a sampling rate of $2 \mathrm{~s}$. The resulting time course of the resting baseline condition was subtracted from the time course of the task condition in order to remove the decreasing signal from the previous trial (Burock et al., 1998). From these time courses, three parameters were extracted: (a) time-to-onset: marks the point along the time course where the BOLD response starts rising steeply from the baseline, (b) time-to-peak: marks the point where the signal flattens out again, and (c) time-to-maximum: marks the point where the signal reaches its maximum (for details, see Neumann et al., 2003). Reliably determining onsets and peak values in single subject data proofed to be difficult. By averaging 
single-subject time courses across subjects, only unique values for onset, peak, and maximum can be extracted from the resulting grand mean time course. Any information about the variability of the extracted values within the group is lost. The jackknife resampling procedure (Efron, 1981; Miller, 1974) provides an elegant tool to create numerous averaged time courses for the estimation of parameters (see Ruge et al., 2003). Each of the 15 subjects is excluded from the time course averaging once and the parameters are extracted from the averaged time course. The resulting 15 extracted parameters (each omitting a different subject) can then be averaged and used for estimates of standard errors.

\section{Results}

\section{Behavioral results}

Fig. 1 shows the averaged reaction times (RT) for the four similarity levels of the decisions. A significant increase in RT was elicited with increasing similarity of the two presented alternatives (ANOVA with repeated measures; $F(3 / 42)=25.06 ; P<0.001$ ). The RT of the Easy-Level is significantly different from the Medium1-Level (paired $t$ test; $P=0.001$ ), and Medium2-Level differed from the Difficult-Level $(P<0.001)$, but the Medium1Level did not significantly differ from the Medium2-Level $(P=$ 0.498). These results indicate that the decisional effort becomes greater with increasing similarity of the two alternatives and that the manipulation of the similarity was successfully implemented.

\section{Imaging results}

Of primary interest were areas changing their activation parametrically with the variation of the similarity of the two alternatives. The parametric contrast showed a wide ranging network reacting to increasing decisional effort. Fig. 2 and Table 3 report those areas whose level of activation was related significantly to the similarity manipulation. Three regions along the left inferior frontal sulcus (IFS) were significantly activated: the
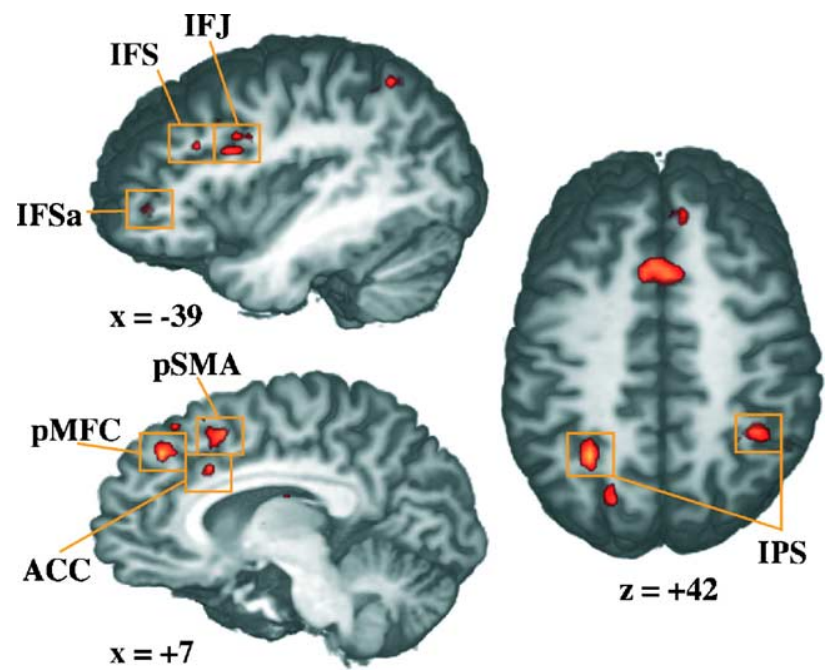

Fig. 2. Activation maps of the parametric modulation of similarity between the alternatives mapped onto an individual brain. $Z$ values were thresholded at $z=3.8$, representing a False Discovery Rate of $P<0.01$.
Table 3

Talairach coordinates, maximum $Z$ value and volume $\left(\mathrm{mm}^{3}\right)$ of the significantly activated areas for the parametric contrast for similarity between alternatives

\begin{tabular}{lccc}
\hline Area & Volume & $Z$ max & $\begin{array}{c}\text { Talairach } \\
\text { coordinates }\end{array}$ \\
\hline $\begin{array}{l}\text { L/R. presupplementary } \\
\quad \text { motor area (BA 6) }\end{array}$ & 1927 & 5.61 & $4,11,47$ \\
$\begin{array}{l}\text { R. posterior medial } \\
\quad \text { frontal cortex (BA 8) }\end{array}$ & 308 & 4.68 & $7,38,38$ \\
R. anterior cingulate & & & \\
$\quad$ cortex (BA24) & 130 & 4.47 & $8,17,32$ \\
L. inferior frontal junction area & 597 & 4.43 & $-34,2,30$ \\
L. inferior frontal sulcus & 127 & 4.32 & $-41,20,30$ \\
L. anterior inferior frontal sulcus & 118 & 4.35 & $-37,41,6$ \\
R. inferior frontal sulcus & 202 & 4.51 & $43,26,24$ \\
L. anterior insula & 402 & 4.70 & $-26,20,0$ \\
L. intraparietal sulcus & 790 & 4.65 & $-29,-55,41$ \\
R. intraparietal sulcus & 359 & 4.84 & $49,-52,47$ \\
L. caudate & 312 & 4.37 & $-10,5,6$ \\
\hline
\end{tabular}

We used corrected $P$ values with the False Discovery Rate method of at least $P<0.01$ and reported activations had a minimal size of $81 \mathrm{~mm}^{3}$ (3 voxels).

inferior frontal junction area (IFJ), the middle section of the IFS, and the anterior part of the IFS (IFSa). In the right hemisphere, this activation was less prominent and restricted to the middle IFS. Another cluster of activations was found in the medial surface of the prefrontal cortex, namely in the presupplementary motor area (preSMA) bilaterally, in the posterior medial frontal cortex (pMFC), and in the anterior cingulate cortex (ACC). The anterior insula was activated in the left hemisphere only. Further, two distinct regions in the left and right intraparietal sulcus and the left caudate nucleus showed significant activations.

In order to classify the activated regions according to the temporal dynamics, the underlying time courses were extracted and analyzed (see Methods section). For the main activations reported in Table 3, time-to-onset, time-to-peak, and time-to-max were calculated and are reported in Table 4. All three parameters

Table 4

Time-to-onset, time-to-peak, and time-to-maximum values along with the standard error of mean of the main activations reported in Table 3

\begin{tabular}{lccc}
\hline Area & Time-to-onset & Time-to-peak & Time-to-max \\
\hline $\begin{array}{l}\text { L. inferior frontal } \\
\text { junction area }\end{array}$ & $2.6(0.08)$ & $6.7(0.11)$ & $9.8(0.33)$ \\
$\begin{array}{l}\text { L. intraparietal sulcus } \\
\text { L/R. presupplementary }\end{array}$ & $2.9(0.18)$ & $10.0(0.15)$ & $12.0(0.15)$ \\
$\quad$ motor area & & $13.9(0.07)$ & $16.0(0.06)$ \\
L. inferior frontal sulcus & $6.1(0.17)$ & $10.6(0.16)$ & $14.5(0.13)$ \\
R. inferior frontal sulcus & $6.3(0.23)$ & $12.4(0.16)$ & $12.6(0.16)$ \\
R. intraparietal sulcus & $7.6(0.13)$ & $12.7(0.17)$ & $16.0(0.14)$ \\
R. posterior medial & $8.7(0.46)$ & $9.8(0.43)$ & $12.0(0.55)$ \\
$\quad$ frontal cortex (BA 8) & & & \\
L. anterior inferior & $8.9(0.15)$ & $11.9(0.20)$ & $14.1(0.20)$ \\
$\quad$ frontal sulcus & $9.5(0.24)$ & $12.3(0.27)$ & $14.3(0.29)$ \\
$\begin{array}{l}\text { L. anterior insula } \\
\text { L. caudate }\end{array}$ & $11.3(0.34)$ & $14.2(0.23)$ & $16.4(0.20)$ \\
R. anterior cingulate & $11.8(0.27)$ & $14.2(0.22)$ & $16.8(0.15)$ \\
$\quad$ cortex (BA24) & & & \\
\hline
\end{tabular}

The regions are ordered by the time-to-onset value. 
estimated from the time courses varied significantly between regions (ANOVA across regions; onset: $P<0.001$; peak: $P<0.01$; $\max : P<0.05)$. Some regions showed an early increase right from the beginning of the trial (preSMA, IPS, and IFJ), whereas other regions had a delayed signal increase (IFS, pMFC, ACC, anterior insula, and caudate). Fig. 3 shows examples of the extracted time courses for regions with early and late onsets. The left IPS, preSMA, and the IFJ showed a signal increase right from the beginning. The signal of the IPS and preSMA increased steadily, reaching its maximum height after $12 \mathrm{~s}$ and $16 \mathrm{~s}$, respectively It is argued that regions showing a steady increase are related to the integration of information, the process level of decision making. Interestingly, the left IFJ showed an early signal increase but the time-to-peak was reached after $7 \mathrm{~s}$, and the signal remained constant after that. The middle section of the IFS (bilaterally) showed a delayed signal increase after $6 \mathrm{~s}$. The pMFC, the IFSa, and anterior insula displayed signal increases not before $8-10 \mathrm{~s}$. We consider those regions with a delayed onset to be related to the control level of decision making. The ACC and the caudate showed a signal increases only after $11 \mathrm{~s}$, at the moment when the last frame was presented. This indicates that these regions are response related.
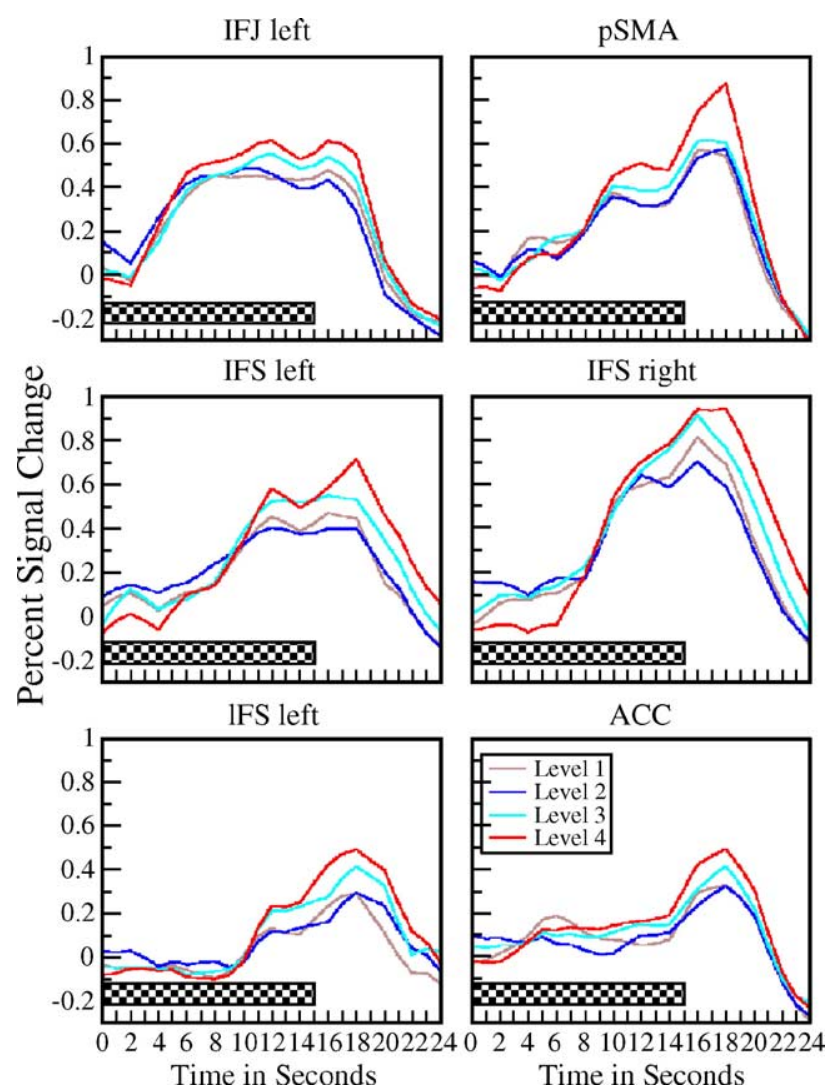

Fig. 3. Group-averaged time courses for 6 specific regions of interest. The gray box indicates the duration of the task. The signal was averaged for each of the 4 levels of similarity, and the resting baseline was subtracted. The preSMA shows an example of an early onset and steady increase, the IFJ left of an early onset and early saturation. The anterior IFS (left and right) is an example for a delayed hemodynamic response, and the anterior IFS and the ACC show a clearly delayed hemodynamic response.

\section{Discussion}

The present findings demonstrate that our experimental task required a distributed neural network in order to decide between competing alternatives (see Fig. 2 and Table 3). The network is predominantly located in the left hemisphere and incorporates inferior frontolateral, parietal, and posterior medial frontal areas as well as the anterior insula and the caudate nucleus. The dominance of the left hemisphere is in accordance with findings of Goldberg and Podell $(1999,2000)$ and Podell et al. (1995) who suggest a hemispheric difference in the frontolateral cortex in relation to similarity processing. Further, Goel and Dolan (2000) showed that the left hemisphere is dominant in processing problems with explicit contextual cues. But one also has to consider that the task was described in a verbal domain, so that this lateralization my result from the predominance of the left hemisphere in verbal processing. Language processing is known to be lateralized to the left hemisphere in most individuals (Binder and Price, 2001; Bookheimer, 2002; Friederici, 2002). Because the nature of our decision making paradigm was highly dependent on linguistic reasoning, it may not be surprising that the results are also strongly lateralized. However, effects of language processing do not differ between the critical conditions in our experiment.

Within this network, areas can be differentiated according to the temporal dynamics of the hemodynamic response: (i) regions with early onset and steady increase, (ii) regions with early onset and early saturation, and (iii) regions with late onset. Regions with early onset and steady increase in signal intensity are the preSMA and regions along the left IPS (see Fig. 2). One could assume that the IPS and preSMA are constantly working on integrating the attribute information and processing additional aspects of the two alternatives and thus show a steadily increasing BOLD response. We argue that these regions subserve the process level of decision making. It has been shown previously (Goel and Grafman, 2000; Smith et al., 1998) that the superior parietal lobe is involved in decision making, related to the integration of unbound information (Prabhakaran et al., 2000). A study by Heekeren et al. (2004) showed that regions along the IPS, and additionally in the frontal eye fields (SEF and FEF) showed greater activations with increasing attentional demand. It appears that the preSMA activation in the present study includes the area of the SEF.

A second group of regions exhibits a delayed onset of the BOLD response. These regions include the left and right IFS, the pMFC, the left insula, as well as the anterior IFS. We suggest that these regions are related to the control level of decision making, i.e., a level which is conceived of as subserving the control of subprocesses. Since this process depends on the process level, it starts when the two alternatives begin to differ. This process is minimal at the beginning of each trial, as no differences occur. But with ongoing trial duration, differences occur and have to be evaluated for significance. This results in delayed signal increases. This interpretation of inferior prefrontal activations is consistent with findings by Paulus et al. (2004) who propose this region to be related to the acquisition of advantageous actions or a moment-tomoment model as proposed by Huettel et al. (2002). The activation in the pMFC corresponds well with findings from Volz et al. (2003, 2004) who showed that this region reflects the degree of uncertainty and is related to decision conflicts, which in the present study increase with increasing similarity.

The anterior insula has been proposed on several occasion to be related to decision making, i.e., in interactional games (Sanfey et 
al., 2003), reward contingencies (O'Doherty et al., 2003), or risktaking aspects and harm avoidance (Paulus et al., 2003b). Activation in the anterior insula has also been associated with several other functions like speech motor control (Ackermann and Riecker, 2004), feeling disgust (Wicker et al., 2003), autonomic arousal (Decety et al., 2004), uncertainty (Volz et al., 2003), cognitive paradigms like the Stroop task (Zysset et al., 2001), or task switching (Derrfuss et al., 2004). The anterior insula appears to be related to risk taking not only in decision making but subserves general processes which are also essential in decision making. In general, the anterior insula may provide a 'gut' feeling aid in the control level of decision making (Paulus et al., 2005) which may be mediated by autonomic states.

A third characteristic time course of the signal could be observed in the left IFJ. Here, the BOLD signal had an early onset but saturated after $6 \mathrm{~s}$. It could be argued that the IFJ is activated from trial onset; however, this initial change in signal is seen only for a short period of time after each presentation of an additional attribute. Hence, the IFJ is not continuously integrating the presented information as the IPS. If the IFJ region had been constantly activated, the signal would have steadily increased, and the signal change would have saturated 15-20 s after onset (Boynton et al., 1996). In the present case, the signal saturates at a much earlier point in time and remains constant afterwards. This result is in accordance with previous studies showing that the IFJ is essential for the activation of task representations, and the presentation of every new attribute makes it necessary to re-initiate the task sets (Brass et al., 2005; Derrfuss et al., 2004; Brass and von Cramon, 2002, 2004).

This dissociation between two levels in decision making (process and control level) corresponds to the model put forward by Shadlen and Newsome (1996, 2001). Their studies with monkeys revealed a first level of information integration and a second stage of information interpretation. These stages were also found for perceptual decision making in human subjects (Heekeren et al., 2004). In the present study, the employed task is based on more complex cognitive processes compared to the perceptual categorization task. We were able to show that corresponding areas in the supplementary motor area and the IPS appear to play an essential role in integrating the perceived information.

Further, the information interpretation appears to be centered in the inferior lateral and medial frontal cortex, and prominent activations were found in the anterior cingulate cortex (the rostral cingulate zone; RCZ) and the left caudate. The RCZ has been reported to be relevant in sorting among conflicting options (Krawczyk, 2002; Ullsperger and von Cramon, 2004). As the time courses revealed, these regions were activated very late, at the time of the actual response. This supports the view that the RCZ is response related and involved in post-decisional processes.

The task used in the present study will allow a wide range of variations to differentiate the involved subprocesses more precisely. For example, if the subject is able to terminate each trial as soon as a decision is reached, the timing of the control level can be further differentiated. The introduction of conflicting alternatives or knock-out attributes allows to differentiate between the implementation of different heuristics. The variation of the presentation rate of the attributes, as well as using more than two alternatives, will help to resolve further the interaction of the process and control level and other subprocesses related to decision making. The task allows the introduction of uncertainty, ambiguity, missing information as well as reward, and by this the investigation of additional processes not tabbed in the presently used version. We see the present study as a first step in establishing this behavioral paradigm in the neuroimaging setup. The complexity of the task might be a limitation for the interpretation of the underlying processes but a necessary one when modeling more realistic decision-making situations. Further studies with the multiattribute decision task will allow to distinguish more subtle processes related to decision making.

\section{Conclusion}

In summary, the current investigation showed that a distributed neural network is involved in solving a rather simple decisionmaking task. This network includes the left and right frontolateral cortex, the superior parietal lobe, the posterior medial frontal cortex, the anterior insula, as well as the caudate nucleus. Whereas the parietal lobe and preSMA are suggested to be related to the integration of information (process level), the lateral prefrontal cortex and the posterior medial frontal cortex are related to the interpretation of this integrated information (control level). This study is a first step in investigating decision making at a more complex level using a more complex but also more realistic task. It proposes a model, as well as a method, to investigate decision making at a more realistic level.

\section{Acknowledgments}

The authors would like to thank Mandy Naumann, Simone Wipper and Annett Wiedemann for their support with acquiring the fMRI-data and Shirley-Ann Rüschemeyer for her helpful comments on the manuscript.

\section{References}

Ackermann, H., Riecker, A., 2004. The contribution of the insula to motor aspects of speech production: a review and a hypothesis. Brain Lang. 89 (2), 320-328.

Aguirre, G.K., Zarahn, E., d'Esposito, M., 1997. Empirical analyses of BOLD fMRI statistics: II. Spatially smoothed data collected under nullhypothesis and experimental conditions. NeuroImage 5, 199-212.

Aschenbrenner, K.M., Albert, D., Schmalhofer, F., 1984. Stochastic choice heuristics. Acta Psychol. 56, 153-166.

Bechara, A., Tranel, B., Damasio, H., Damasio, A.R., 1996. Failure to respond autonomically to anticipated future outcomes following damage to prefrontal cortex. Cereb. Cortex 6, 215-225.

Bechara, A., Damasio, H., Tranel, D., Damasio, A.R., 1997. Deciding advantageously before knowing the advantageous strategy. Science 275, $1293-1295$

Bechara, A., Damasio, H., Damasio, A., 2000. Emotion, decision making and the orbitofrontal cortex. Cereb. Cortex 10 (3), 295-307.

Benjamini, Y., Hochberg, Y., 1995. Controlling the False Discovery Rate: a practical and powerful approach to multiple testing. J. R. Stat. Soc. 57, 289-300.

Benjamini, Y., Yekutieli, D., 2001. The Control of the False Discovery Rate in multiple testing under dependency. Technical report, School of Mathematical Sciences. Tel Aviv University, http://www.math. tau.ac.il/benja/

Binder, J., Price, C., 2001. Functional Neuroimaging of Language. In: Cabeza, R., Kingstone, A. (Eds.), Handbook of Functional Neuroimaging of Cognition. Chapter 7. A Bradford Book, Cambridge, M.A., pp. $187-252$. 
Blackwood, N., Ffytche, D., Simmons, A., Bentall, R., Murray, R., Howard, R., 2004. The cerebellum and decision making under uncertainty. Brain Res. Cogn. Brain Res. 20 (1), 46-53.

Bookheimer, S., 2002. Functional MRI of language: new approaches to understanding the cortical organization of semantic processing. Annu. Rev. Neurosci. 25, 151-188.

Boynton, G.M., Engel, S.A., Glover, G.H., Heeger, D.J., 1996. Linear systems analysis of 20 functional magnetic resonance imaging in human V1. J. Neurosci. 16 (13), 4207-4221.

Brass, M., von Cramon, D., 2002. The role of the frontal cortex in task preparation. Cereb. Cortex 12 (9), 908-914.

Brass, M., von Cramon, D., 2004. Decomposing components of task preparation with functional magnetic resonance imaging. J. Cogn. Neurosci. 16 (4), 609-620.

Brass, M., Derrfuss, J., Forstmann, B., von Cramon, D.Y., 2005. The role of the inferior frontal junction area in cognitive control. Trends Cogn. Sci. 9, 314-316

Burock, M., Buckner, R., Woldorff, M., Rosen, B., Dale, A., 1998. Randomized event-related experimental designs allow for extremely rapid presentation rates using functional MRI. NeuroReport 9 (16), $3735-3739$.

Bush, G., Vogt, B., Holmes, J., Dale, A., Greve, D., Jenike, M., Rosen, B., 2002. Dorsal anterior cingulate cortex: a role in reward-based decision making. Proc. Natl. Acad. Sci. U. S. A. 99 (1), 523-528.

Decety, J., Jackson, P., Sommerville, J., Chaminade, T., Meltzoff, A., 2004. The neural bases of cooperation and competition: an fMRI investigation. NeuroImage 23 (2), 744-751.

Derrfuss, J., Brass, M., von Cramon, D., 2004. Cognitive control in the posterior frontolateral cortex: evidence from common activations in task coordination, interference control, and working memory. NeuroImage 23 (2), 604-612.

Efron, B., 1981. Nonparametric estimates of standard error: the jackknife, the bootstrap, and other methods. Biometrika 68, 589-599.

Elliott, R., Dolan, R., 1998. Activation of different anterior cingulate foci in association with hypothesis testing and response selection. Neurolmage $8,17-29$.

Ford, J.K., Schmitt, N., Schechtman, S.L., Hults, B.M., Doherty, M.L., 1989. Process tracing methods: contributions, problems, and neglected research questions. Organizational Behavior and Human Decision Processes 43, 75-117.

Friederici, A., 2002. Towards a neural basis of auditory sentence processing. Trends Cogn. Sci. 6 (2), 78-84.

Friston, K.J., Holmes, A.P., Worsley, K.J., Poline, J.-B., Frith, C.D., Frackowiak, R.W.J., 1995. Statistical parametric maps in functional imaging: a general linear approach. Hum. Brain Mapp. 2, 189-210.

Gehring, W., Willoughby, A., 2002. The medial frontal cortex and the rapid processing of monetary gains and losses. Science 295 (5563), $2279-2282$.

Genovese, C., Lazar, N., Nichols, T., 2002. Thresholding of statistical maps in functional neuroimaging using the False Discovery Rate. NeuroImage 15 (4), 870-878.

Glover, G.H., 1999. Deconvolution of impulse response in event-related BOLD fMRI. NeuroImage 9, 416-429.

Goel, V., Dolan, R.J., 2000. Anatomical segregation of component processes in an inductive inference task. J. Cogn. Neurosci. 12, $110-119$.

Goel, V., Grafman, J., 2000. Role of the right prefrontal cortex in illstructured planning. Cogn. Neuropsychol. 17 (5), 415-436.

Goel, V., Grafman, J., Tajik, J., Gana, S., Danto, D., 1997. A study of the performance of patients with frontal lobe lesions in a financial planning task. Brain 120, 1805-1822.

Goldberg, E., Podell, K., 1999. Adaptive versus veridical decision making and the frontal lobes. Conscious. Cogn. 8, 364-377.

Goldberg, E., Podell, K., 2000. Adaptive decision making, ecological validity, and the frontal lobes. J. Clin. Exp. Neuropsychol. 22 (1), $56-68$.

Haruno, M., Kuroda, T., Doya, K., Toyama, K., Kimura, M., Samejima,
K., Imamizu, H., Kawato, M., 2004. A neural correlate of rewardbased behavioral learning in caudate nucleus: a functional magnetic resonance imaging study of a stochastic decision task. J. Neurosci. 24 (7), $1660-1665$.

Heekeren, H., Wartenburger, I., Schmidt, H., Schwintowski, H., Villringer, A., 2003. An fMRI study of simple ethical decision-making. NeuroReport 14 (9), 1215-1219.

Heekeren, H., Marrett, S., Bandettini, P., Ungerleider, L., 2004. A general mechanism for perceptual decision-making in the human brain. Nature 431 (7010), 859-862.

Heekeren, H., Wartenburger, I., Schmidt, H., Prehn, K., Schwintowski, H., Villringer, A., 2005. Influence of bodily harm on neural correlates of semantic and moral decision-making. NeuroImage 24 (3), 887-897.

Holmes, A.P., Friston, K.J., 1998. Generalisability, random effects and population inference. NeuroImage 7, 754 (Suppl.)

Huber, O., 1989. Information-processing operators in decision making. In: Montgomery, H., Svenson, O. (Eds.), Process and structure in human decision making S. 321. Wiley, Chichester, NY.

Huber, O., 2002. Risk defusing behavior. In: Derigs, U. (Ed.), Optimization and operations research, Band 6.5 of the Encyclopedia of Life Support Systems (EOLSS). Eolss Publisher, Oxford. (http://www.eolss.ne, Bd.)

Huettel, S., Mack, P., McCarthy, G., 2002. Perceiving patterns in random series: dynamic processing of sequence in prefrontal cortex. Nat. Neurosci. 5 (5), $485-490$.

Kim, J., Shadlen, M., 1999. Neural correlates of a decision in the dorsolateral prefrontal cortex of the macaque. Nat. Neurosci. 2 (2), $176-185$

Krawczyk, D., 2002. Contributions of the prefrontal cortex to the neural basis of human decision making. Neurosci. Biobehav. Rev. 26 (6), $631-664$

Lohmann, G., Muller, K., Bosch, V., Mentzel, H., Hessler, S., Chen, L., Zysset, S., von Cramon, D.Y., 2001. Lipsia-A new software system for the evaluation of functional magnetic resonance images of the human brain. Comput. Med. Imaging Graph. 25 (6), 449-457.

Miller, R.G., 1974. The jackknife: a review. Biometrika 61, 1-15.

Moll, J., de Oliveira-Souza, R., Moll, F., Ignacio, F., Bramati, I., CaparelliDaquer, E., Eslinger, P., 2005. The moral affiliations of disgust: a functional MRI study. Cogn. Behav. Neurol. 18 (1), 6878.

Neumann, J., Lohmann, G., Zysset, S., von Cramon, D.Y., 2003. Withinsubject variability of BOLD response dynamics. NeuroImage 19 (3), $784-796$.

Norris, D.G., 2000. Reduced power multi-slice MDEFT imaging. J. Magn. Reson. Imaging 11, 445-451.

O’Doherty, J.P., Critchley, H.D., Deichmann, R., Dolan, R.J., 2003. Dissociating valence of outcome from behavioral control in human orbital and ventral prefrontal cortices. J. Neurosci. 23, 7931-7939.

Paulus, M.P., Hozack, N., Zauscher, B., McDowell, J.E., Frank, L., Brown, G.G., Braff, D.L., 2001. Prefrontal, parietal, and temporal cortex networks underlie decision-making in the presence of uncertainty. NeuroImage 13 (1), 91-100

Paulus, M., Hozack, N., Frank, L., Brown, G., Schuckit, M., 2003a. Decision making by methamphetamine-dependent subjects is associated with error-rate-independent decrease in prefrontal and parietal activation. Biol. Psychiatry 53 (1), 65-74.

Paulus, M., Rogalsky, C., Simmons, A., Feinstein, J., Stein, M., 2003 b. Increased activation in the right insula during risk-taking decision making is related to harm avoidance and neuroticism. NeuroImage 19 (4), 1439-1448.

Paulus, M.P., Feinstein, J.S., Tapert, S.F., Liu, T.T., 2004. Trend detection via temporal difference model predicts inferior prefrontal cortex activation during acquisition of advantageous action selection. NeuroImage 21, 733-743 (24).

Paulus, M.P., Feinstein, J.S., Leland, D., Simmons, A.N., 2005. Superior temporal gyrus and insula provide response and outcome-dependent information during assessment and action selection in a decisionmaking situation. NeuroImage 25 (2), 607-615. 
Payne, J.W., Bettman, J.R., Johnson, E.J., 1993. The Adaptive Decision Maker. Cambridge Univ. Press, Cambridge.

Podell, K., Lovell, M., Zimmermann, M., Goldberg, E., 1995. The cognitive bias task and lateralized frontal lobe functions in males. J. Neuropsychiatry 7, 491-501.

Prabhakaran, V., Smith, J., Desmond, A.L., Glover, G., Gabrieli, J.D.E., 1997. Neural substrates of fluid reasoning: an fMRI study of neocortical activation during performance of the Raven's progressive matrices test. Cogn. Psychol. 33, 43-63.

Prabhakaran, V., Narayanan, K., Zhao, Z., Gabrieli, J., 2000. Integration of diverse information in working memory within the frontal lobe. Nat. Neurosci. 3 (1), 85-90.

Reddi, B., 2001. Decision making: the two stages of neuronal judgement. Curr. Biol. 11 (15), R603-R606.

Rieskamp, J., Hoffrage, U., 1999. When do people use simple heuristics, and how can we tell? In: Gigerenzer, G., Todd, P.M., ABC Research Group (Eds.), Simple Heuristics That Make Us Smart. Oxford Univ. Press, New York. (S. 141-167).

Rogers, R., Ramnani, N., Mackay, C., Wilson, J., Jezzard, P., Carter, C., Smith, S., 2004. Distinct portions of anterior cingulate cortex and medial prefrontal cortex are activated by reward processing in separable phases of decision-making cognition. Biol. Psychiatry 55 (6), 594-602.

Ruge, H., Brass, M., Lohmann, G., von Cramon, D.Y.C., 2003. Eventrelated analysis for event types of fixed order and restricted spacing by temporal quantification of trial-averaged fMRI time course. J. Magn. Reson. Imaging 18, 599-607.

Sakagami, M., Tsutsui, K., 1999. The hierarchical organization of decision making in the primate prefrontal cortex. Neurosci. Res. 34 (2), 7989.

Sanfey, A., Rilling, J., Aronson, J., Nystrom, L., Cohen, J., 2003. The neural basis of economic decision-making in the Ultimatum Game. Science 300 (5626), 1755-1758.

Schall, J., 2002. Decision making: neural correlates of response time. Curr. Biol. 12 (23), R8001.

Shadlen, M., Newsome, W., 1996. Motion perception: seeing and deciding. Proc. Natl. Acad. Sci. U. S. A. 93 (2), 628-633.

Shadlen, M., Newsome, W., 2001. Neural basis of a perceptual decision in the parietal cortex (area LIP) of the rhesus monkey. J. Neurophysiol. 86 (4), 1916-1936.

Slovic, P., Fischhoff, B., Lichtenstein, S., 1977. Behavioral decision theory. Annu. Rev. Psychol. 28, 1-39.
Smith, E.E., Jonides, J., Marshuetz, C., Koeppe, R.A., 1998. Components of verbal working memory: evidence from Neuroimaging. Proc. Natl. Acad. Sci. U. S. A. 95, 876-882.

Svenson, O., 1979. Process description of decision making. Organ. Behav. Hum. Perform. 23, 86-112.

Svenson, O., 1993. Differentiation and consolidation theory of human decision making: a frame of reference for the study of pre- and postdecision processes. In: Huber, O., Mumpower, J., Van der Pligt, J., Koele, P. (Eds.), Current themes in Psychological Decision Research. North Holland, Amsterdam. (S. 143 168).

Talairach, J., Tournoux, P., 1988. Co-Planar Stereotaxic Atlas of the Human Brain. Thieme Medical Publisher Inc, Stuttgart.

Turk, D., Banfield, J., Walling, B., Heatherton, T., Grafton, S., Handy, T., Gazzaniga, M., Macrae, C., 2004. From facial cue to dinner for two: the neural substrates of personal choice. NeuroImage 22 (3), $1281-1290$

Ugurbil, K., Garwood, M., Ellermann, J., Hendrich, K., Hinke, R., Hu, X., Kim, S.-G., Menon, R., Merkle, H., Ogawa, S., Salmi, R., 1993. Imaging at high magnetic fields: initial experiences at 4t. Magn. Reson. Q. 9, 259.

Ullsperger, M., von Cramon, D., 2004. Decision making, performance and outcome monitoring in frontal cortical areas. Nat. Neurosci. 7 (11), $1173-1174$.

Volz, K., Schubotz, R., von Cramon, D., 2003. Predicting events of varying probability: uncertainty investigated by fMRI. NeuroImage 19 (2 Pt. 1), $271-280$.

Volz, K., Schubotz, R., von Cramon, D., 2004. Why am I unsure? Internal and external attributions of uncertainty dissociated by fMRI. NeuroImage 21 (3), 84857.

Wicker, B., Keysers, C., Plailly, J., Royet, J., Gallese, V., Rizzolatti, G., 2003. Both of us disgusted in My insula: the common neural basis of seeing and feeling disgust. Neuron 40 (3), 655-664.

Worsley, K., Friston, K., 1995. Analysis of fMRI time-series revisitedAgain. NeuroImage 2, 173-181.

Zarahn, E., Aguirre, G.K., d'Esposito, M., 1997. Empirical analyses of BOLD fMRI statistics: I. Spatially unsmoothed data collected under null-hypothesis conditions. NeuroImage 5, 179-197.

Zysset, S., Müller, K., Lohmann, G., von Cramon, D.Y., 2001. Color-word matching Stroop task: separating interference and response conflict. NeuroImage $13,29-36$. 